\title{
Bicaudal-D uses a parallel, homodimeric coiled coil with heterotypic registry to coordinate recruitment of cargos to dynein
}

\author{
Yang Liu, ${ }^{1,6,7}$ Hannah K. Salter, ${ }^{1,6}$ Andrew N. Holding, ${ }^{1}$ Christopher M. Johnson, ${ }^{2}$ Elaine Stephens, ${ }^{1,8}$ \\ Peter J. Lukavsky, ${ }^{3}$ John Walshaw, ${ }^{4,5}$ and Simon L. Bullock ${ }^{1,9}$ \\ ${ }^{1}$ Division of Cell Biology, ${ }^{2}$ Division of Protein Nucleic Acid Chemistry, MRC Laboratory of Molecular Biology, Cambridge CB2 \\ 0QH, United Kingdom; ${ }^{3}$ Central European Institute of Technology, Masaryk University, Brno CZ-62500, Czech Republic; \\ ${ }^{4}$ Institute of Food Research, Norwich Research Park, Norwich NR4 7UA, United Kingdom; ${ }^{5}$ School of Computing Sciences, \\ University of East Anglia, Norwich NR4 7TJ, United Kingdom
}

\begin{abstract}
Cytoplasmic dynein is the major minus end-directed microtubule motor in eukaryotes. However, there is little structural insight into how different cargos are recognized and linked to the motor complex. Here we describe the $2.2 \AA$ resolution crystal structure of a cargo-binding region of the dynein adaptor Bicaudal-D (BicD), which reveals a parallel coiled-coil homodimer. We identify a shared binding site for two cargo-associated proteins-Rab6 and the RNA-binding protein Egalitarian (Egl)-within a region of the BicD structure with classical, homotypic core packing. Structure-based mutagenesis in Drosophila provides evidence that occupancy of this site drives association of BicD with dynein, thereby coupling motor recruitment to cargo availability. The structure also contains a region in which, remarkably, the same residues in the polypeptide sequence have different heptad registry in each chain. In vitro and in vivo analysis of a classical Drosophila dominant mutation reveals that this heterotypic region regulates the recruitment of dynein to BicD. Our results support a model in which the heterotypic segment is part of a molecular switch that promotes release of BicD autoinhibition following cargo binding to the neighboring, homotypic coiled-coil region. Overall, our data reveal a pivotal role of a highly asymmetric coiled-coil domain in coordinating the assembly of cargo-motor complexes.
\end{abstract}

[Keywords: Bicaudal-D; coiled coil; dynein; cargo binding; crystal structure; Drosophila.]

Supplemental material is available for this article.

Received December 17, 2012; revised version accepted May 1, 2013.

Cytoplasmic dynein is the major minus end-directed microtubule motor in the eukaryotic kingdom and thus plays critical roles in the sorting of diverse cellular cargos and pathogens (Kardon and Vale 2009). Dynein is an $~ 1.5-$ MDa complex containing two copies of a heavy chain (which harbors the force-generating AAA ATPase motor domain) as well as intermediate, light intermediate, and light chain subunits. Recent structural work has provided detailed insights into the mechanochemistry of the motor domain (Carter 2013). However, much less is known about how dynein recognizes specific cargos and how the assembly of cargo-motor complexes is orchestrated.

\footnotetext{
${ }^{6}$ These authors contributed equally to this work.

Present addresses: ${ }^{7}$ MRC Cancer Cell Unit, Cambridge CB2 0XZ, United Kingdom; ${ }^{8}$ Blue Stream Laboratories, 763 Concord Ave, Cambridge, Massachusetts 02138, USA.

${ }^{9}$ Corresponding author

E-mail sbullock@mrc-lmb.cam.ac.uk

Article published online ahead of print. Article and publication date are online at http://www.genesdev.org/cgi/doi/10.1101/gad.212381.112.
}

The Bicaudal-D (BicD) protein, which is predicted to contain extensive $\alpha$-helical coiled-coil stretches (Supplemental Fig. S1), is one of a small number of proteins implicated to date in the linkage of cargos to the dynein complex (Kardon and Vale 2009). BicD uses features within its $\mathrm{N}$-terminal half (containing predicted coiledcoil segment 1 [CC1] and part of $\mathrm{CC} 2$ ) to associate with dynein and its accessory complex, dynactin (Hoogenraad et al. 2001, 2003; Splinter et al. 2012), with the C-terminal third of BicD (containing CC3) interacting with various adaptor proteins on cellular components and pathogens (Supplemental Fig. S1 and citations therein).

The two best-characterized cargo adaptors for BicD are the small G protein Rab6 and the noncanonical RNAbinding protein Egalitarian (Egl). Rab6 in its GTP-bound state binds to the C-terminal region of Drosophila BicD and its closely related mammalian counterparts, BICD1 and BICD2, as well as a BicD-related protein (BICDR-1) (Matanis et al. 2002; Short et al. 2002; Coutelis and Ephrussi 2007; Januschke et al. 2007; Schlager et al. 
2010). These interactions are important for promoting the dynein-dependent sorting of the Golgi-derived vesicles to which Rab6 ${ }^{\text {GTP }}$ is bound (Matanis et al. 2002; Coutelis and Ephrussi 2007; Januschke et al. 2007; Schlager et al. 2010). The interaction of Egl with the C-terminal region of BicD mediates a functionally important link between dynein-dynactin complexes and asymmetrically localized mRNAs during Drosophila oogenesis and embryogenesis (Navarro et al. 2004; Dienstbier et al. 2009).

Structural insights into the features in the BicD $\mathrm{C}$-terminal region that mediate recognition of cargo adaptors are not available. It is also not known whether this region of BicD binds different partners through discrete binding sites-as is the case for certain other coiled-coil proteins involved in trafficking (e.g., see Shiba et al. 2006; Rosing et al. 2007; Schweizer Burguete et al. 2008; Sinka et al. 2008)-or through a single, multifunctional site. Resolving this issue is important, as it would shed light on how the sorting of different cargos is orchestrated and regulated within cells.

Interestingly, the role of the C-terminal region of BicD is not just restricted to cargo binding. Artificially tethering a heterologous cargo to just the $\mathrm{N}$-terminal two-thirds of mammalian or Drosophila BicD proteins (containing $\mathrm{CC} 1$ and $\mathrm{CC} 2$ ) gives much more robust dynein-dependent targeting than when tethering to the full-length BicD protein is performed (Hoogenraad et al. 2003; Dienstbier et al. 2009; Splinter et al. 2012). It has also been shown that CC3 binds to more N-terminal BicD sequences, although it has not been resolved whether CC2 (as suggested by structural electron microscopy) (Stuurman et al. 1999) or CC1 (as suggested by yeast two-hybrid studies) (Hoogenraad et al. 2001) is the predominant site of interaction. These and other observations indicate that the C-terminal region of BicD participates in autoinhibitory interactions with $\mathrm{N}$-terminal $\mathrm{CC} 1$ and/or CC2 sequences, thereby attenuating their binding to dynein (Hoogenraad et al. 2001, 2003; Splinter et al. 2012). It is possible that the $\mathrm{C}$-terminal region inhibits the binding of the $\mathrm{N}$-terminal sequences to the motor in both the cargo-bound and -unbound states. Alternatively, binding of a cargo adaptor to the C-terminal region could abrogate the autoinhibitory interaction (as proposed by Hoogenraad et al. 2001, 2003). This second model is attractive, as it would allow coupling between the availability of cargo and the ability of BicD's $\mathrm{N}$-terminal sequences to associate with the motor complex. However, this scenario has not been tested directly, and the molecular and structural events that could contribute to such a mechanism are unclear.

A further, long-standing mystery concerning BicD proteins is the molecular basis of classical dominant mutations in the Drosophila gene. Embryos laid by mothers heterozygous for these mutations (E224K or F684I) develop anterior patterning defects, including, in some instances, replacement of anterior structures with posterior structures (these striking double-abdomen embryos give rise to the name of the protein; bicaudal means "two-tailed") (Mohler and Wieschaus 1986; Wharton and Struhl 1989). The BicD-dominant phenotypes are associated with anterior mislocalization of a portion of the mRNA pool en- coding the posterior determinant Oskar (Osk), which is initiated during oogenesis (Ephrussi et al. 1991; Kim-Ha et al. 1991). Genetic interaction experiments indicate that the anterior accumulation of osk mRNA in the mutant results from an increase in Egl- and dynein-dependent localization to the minus ends of microtubules at the expense of plus end-directed, kinesin-1-dependent localization to the posterior cortex (Mohler and Wieschaus 1986; Mach and Lehmann 1997; Brendza et al. 2000; Navarro et al. 2004; Zimyanin et al. 2008). Thus, elucidating the molecular consequences of these mutations could provide valuable insights into how cargo-motor complexes are assembled and operate in vivo.

In this study, we determine the $2.2 \AA$ resolution structure of a region of CC3 of Drosophila BicD containing features important for binding to Rab6 ${ }^{\mathrm{GTP}}$ and $\mathrm{Egl}$ as well as the F684 residue that is mutated in the classical dominant allele. The structure reveals a parallel, homodimeric coiled coil that exhibits a remarkable degree of asymmetry. We identify a shared binding site for both Rab6 ${ }^{\text {GTP }}$ and Egl, which is present in two copies on the opposing faces of a region of coiled coil with canonical, homotypic core packing. Remarkably, the remainder of the coiled-coil structure has heterotypic packing. This arrangement is associated with the highly unusual presence of three bulky aromatic residues at core positions on each chain, one of which is F684. In vitro and in vivo experiments support a model in which the heterotypic coiled-coil region participates in a molecular switch that stimulates association of BicD with dynein upon cargo binding to the adjacent, homotypic segment.

\section{Results}

Drosophila BicD ${ }^{656-745}$ binds Rab6 ${ }^{\text {GTP }}$ with 2:2 stoichiometry and forms a highly asymmetric, parallel coiled coil

To gain structural insights into recognition of cargos by BicD, we sought to solve the crystal structure of amino acids $656-745$ of the Drosophila protein, which exhibited high solubility and purity compared with other CC3 fragments tested. A comparison of the results of previous truncation studies suggests that this sequence, which is very highly conserved in mammalian BICD1 and BICD2 (Schlager et al. 2010), contains features sufficient for binding of BicD to Rab6 ${ }^{\text {GTP }}$ and potentially a variety of other dynein cargos (Supplemental Fig. S1). This region also contains the phenylalanine residue at position 684 that is mutated to isoleucine in the classical dominant allele $B i c D^{1}$ (also known as BicD ${ }^{71.34}$ ) (Wharton and Struhl 1989).

Consistent with previous evidence from ultracentrifugation that the full-length molecule is dimeric (Stuurman et al. 1999), size exclusion chromatography coupled to multiangle light scattering (SEC-MALS) revealed that BicD $^{656-745}$ forms dimers (Fig. 1A). Circular dichroism spectroscopy of this protein suggested that these dimers adopt a coiled-coil structure (Supplemental Fig. S2A). We confirmed that BicD ${ }^{656-745}$ associates with Rab6 ${ }^{\mathrm{GTP}}$ in gel filtration experiments (here and elsewhere, Rab6 ${ }^{\mathrm{GTP}}$ re- 
A
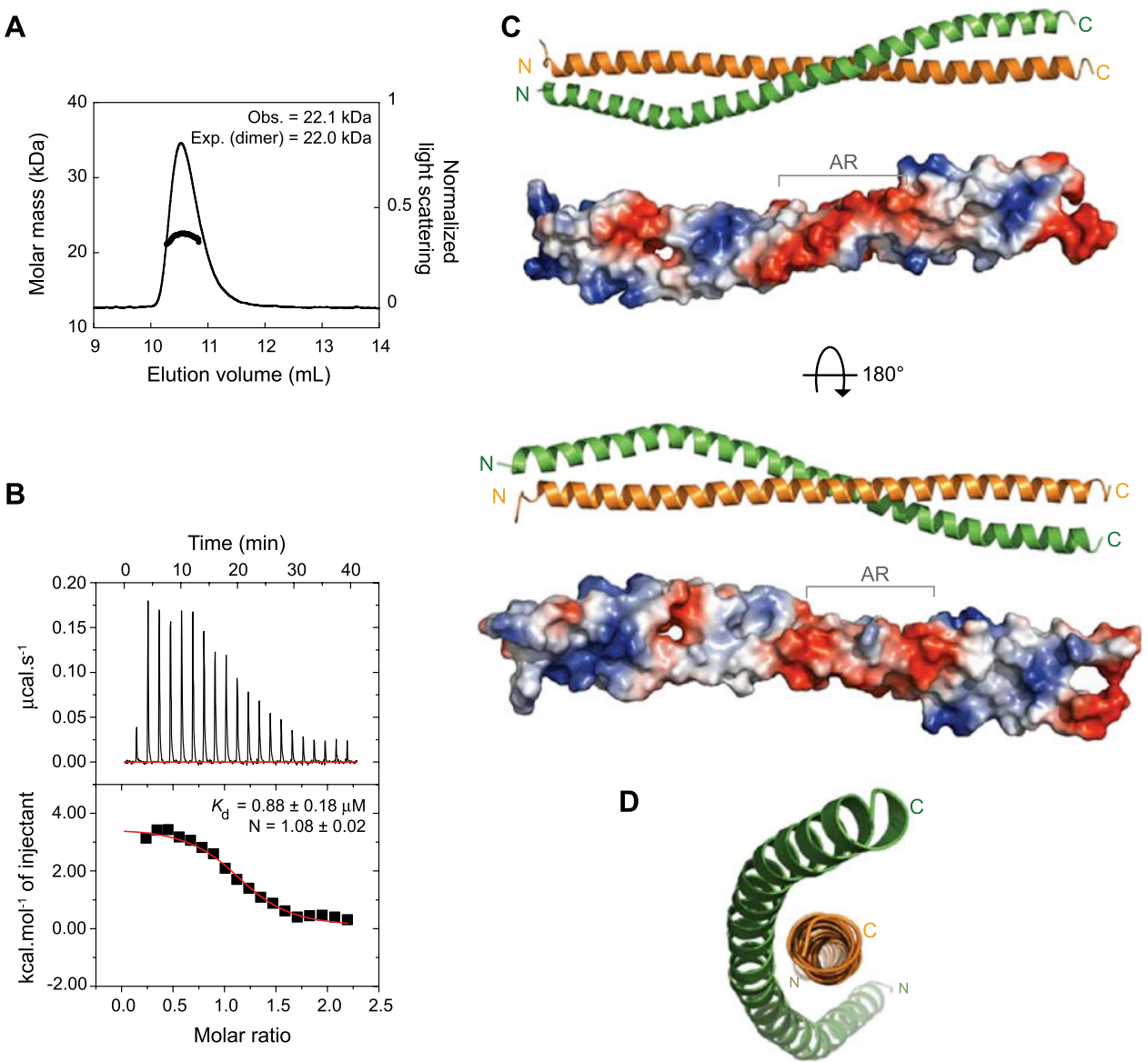

Figure 1. Characterization of $\mathrm{BicD}^{656-745}$ and determined structure. $(A)$ SEC-MALS reveals that recombinant BicD ${ }^{656-745}$ forms a dimer. (Obs.) Mean observed molar mass; [Exp. (dimer)], expected molar mass for a dimer. (B) ITC measurements at $15^{\circ} \mathrm{C}$ of BicD ${ }^{656-745}$ $(29 \mu \mathrm{M})$ titrated with increasing amounts of Rab6 ${ }^{\mathrm{GTP}}$ (up to $\left.300 \mu \mathrm{M}\right)$. Data were fitted to a one-site model. $\left(K_{d}\right)$ Dissociation constant; (N) stoichiometric ratio (value of 1 corresponds to two Rab6 ${ }^{\mathrm{GTP}}$ molecules per BicD dimer). $\Delta \mathrm{H}$ and $\Delta \mathrm{S}$ were $3.0 \pm 0.1 \mathrm{kcal} / \mathrm{mol}$ and $40 \pm 8 \mathrm{cal} / \mathrm{mol} / \mathrm{deg}$, respectively. $(C)$ Overall arrangement and surface representation (electrostatic potential [ $\pm 5.9 \mathrm{kT} / \mathrm{e}] \mathrm{coded}$ in blue [positive] and red [negative]) of the BicD ${ }^{656-745}$ dimer. (AR) Anionic region. The A/D dimer is shown in this and other figures ([orange] A chain; [green] D chain). The B/C dimer forms a highly similar structure (Supplemental Fig. S3A). A small number of residues at the $\mathrm{N}$ and $\mathrm{C}$ termini of the $\mathrm{BicD}^{656-745}$ polypeptide did not show defined electron density and were omitted from the model (Supplemental Fig. S3 legend). (D) End-on view of the dimer, illustrating the marked difference in the degree of supercoiling of the two chains.

fers to the constitutively active Q71L Drosophila protein purified in the presence of GTP or a nonhydrolyzable GTP analog) (Supplemental Fig. S2B). Isothermal titration calorimetry (ITC) — in which a fixed amount of BicD ${ }^{656-745}$ dimer was titrated with increasing concentrations of Rab6 ${ }^{\mathrm{GTP}}$-demonstrated an equimolar Rab6 ${ }^{\mathrm{GTP}}: \mathrm{BicD}$ interaction with a $K_{\mathrm{d}}$ of $\sim 0.9 \mu \mathrm{M}$ (Fig. 1B). Our observations using ITC are consistent with those from a previous study investigating the interaction of CC3 sequences of mammalian BICD2 and Rab6a ${ }^{\text {GTP }}$ (Bergbrede et al. 2009). The equimolar interaction demonstrates that a single BicD $^{656-745}$ dimer binds two copies of Rab6 ${ }^{\mathrm{GTP}}$.

BicD ${ }^{656-745}$ crystallized with two equivalent dimers in the asymmetric unit in spacegroup $P G_{1}$ (Table 1; Supplemental Fig. S3A). The structure was determined by the multiwavelength anomalous dispersion method using selenomethionine-substituted protein. The initial model was built from an untwinned data set with 3.3 A resolution and refined to $2.2 \AA$ with a twinned data set to a free $R$ factor of $25 \%$ (Table 1; Supplemental Fig. S3B,C). The structure of each dimer reveals two extended $\alpha$ helices that form a parallel, left-handed coiled coil that is $\sim 120 \AA$ long (Fig. 1C). There is a pronounced negatively charged surface potential between residues $696 \mathrm{E}$ and $715 \mathrm{E}$, with a mixture of predominantly neutral and positively charged residues elsewhere (Fig. 1C). In contrast to classical homodimeric coiled coils, the two BicD chains produce a highly asymmetric structure. One chain is very straight, with the second chain wrapped around it in a highly supercoiled fashion (Fig. 1C,D). This asymmetry is associated with a remarkable degree of axial stagger of the two chains, particularly in the $\mathrm{N}$-terminal half of the structure (Supplemental Fig. S4). Although irregular parallel coiled-coil dimers have been reported previously (e.g., see Brown 2006; Sato et al. 2007b), we are not aware of one with asymmetry as pronounced as that found in the BicD structure. 
Liu et al.

Table 1. X-ray data collection and refinement statistics

\begin{tabular}{|c|c|c|c|c|}
\hline & \multirow{2}{*}{$\begin{array}{c}\text { Refinement } \\
\text { Peak (twinned) }\end{array}$} & \multicolumn{3}{|c|}{ Phasing (selenium MAD) } \\
\hline & & Peak & Inflection & High energy remote \\
\hline \multicolumn{5}{|l|}{ Data collection } \\
\hline Wavelength & $0.98055 \AA$ & $0.98027 \AA$ & $0.98043 \AA$ & $0.96878 \AA$ \\
\hline Space group & $P 6_{1}$ & $P 6_{1}$ & $P 6_{1}$ & $P 6_{1}$ \\
\hline \multicolumn{5}{|l|}{ Cell dimensions } \\
\hline$a, b, c$ & 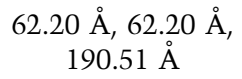 & $\begin{array}{c}66.26 \AA, 66.26 \AA \\
197.24 \AA\end{array}$ & $\begin{array}{c}66.29 \AA, 66.29 \AA \\
197.16 \AA\end{array}$ & $\begin{array}{c}66.14 \AA \text { А, } 66.14 \AA \text { A } \\
197.39 \AA\end{array}$ \\
\hline$\alpha, \beta, \gamma$ & $90^{\circ}, 90^{\circ}, 120^{\circ}$ & $90^{\circ}, 90^{\circ}, 120^{\circ}$ & $90^{\circ}, 90^{\circ}, 120^{\circ}$ & $90^{\circ}, 90^{\circ}, 120^{\circ}$ \\
\hline Resolution & $2.2 \AA$ & $3.3 \AA$ & $3.3 \AA$ & $3.3 \AA$ \\
\hline$R_{\text {merge }}$ & $16.7 \%(102.5 \%)$ & $6.8 \%(64.6 \%)$ & $6.4 \%(54.8 \%)$ & $6.8 \%(65.7 \%)$ \\
\hline$I / \sigma[I]$ & $18.0(4.1)$ & $19.5(4.0)$ & $20.5(4.6)$ & $18.4(4.1)$ \\
\hline Completeness & $100 \%(100 \%)$ & $93.1 \%(80.2 \%)$ & $93.2 \%(81.1 \%)$ & $99.9 \%(100 \%)$ \\
\hline Redundancy & $34.7(34.2)$ & $12.1(13.0)$ & $12.0(12.9)$ & $12.1(13.1)$ \\
\hline \multicolumn{5}{|l|}{ Refinement } \\
\hline Resolution & \multicolumn{4}{|c|}{$46.89 \AA-2.18 \AA(2.231 \AA-2.175 \AA)$} \\
\hline Number of reflections & \multicolumn{4}{|c|}{$20073(1492)$} \\
\hline$R_{\text {work }} / R_{\text {free }}$ & \multicolumn{4}{|c|}{$0.201 / 0.25(0.192 / 0.265)$} \\
\hline \multicolumn{5}{|l|}{ Number of atoms } \\
\hline Protein & \multicolumn{4}{|c|}{2572} \\
\hline Water & \multicolumn{4}{|c|}{107} \\
\hline \multicolumn{5}{|l|}{ Average $B$ factors } \\
\hline Protein & \multicolumn{4}{|c|}{36.71} \\
\hline Water & \multicolumn{4}{|c|}{39.2} \\
\hline \multicolumn{5}{|l|}{ RMS deviations } \\
\hline Bond lengths & \multicolumn{4}{|c|}{$0.016 \AA$} \\
\hline Bond angles & \multicolumn{4}{|c|}{$2.16^{\circ}$} \\
\hline \multicolumn{5}{|l|}{ Ramachandran plot } \\
\hline Most favored regions & \multicolumn{4}{|c|}{$97.5 \%$} \\
\hline Additional allowed regions & \multicolumn{4}{|c|}{$1.9 \%$} \\
\hline Generously allowed regions & \multicolumn{4}{|c|}{$0.0 \%$} \\
\hline Disallowed regions & \multicolumn{4}{|c|}{$0.6 \%$} \\
\hline
\end{tabular}

Numbers in parentheses refer to statistics for the highest-resolution bin.

The same residues in the N-terminal half of each BicD chain simultaneously adopt different heptad positions in the coiled coil

In order to gain further insights into the basis of the asymmetry of the BicD homodimer, we examined its coiled-coil architecture in detail. Coiled coils contain a heptad repeat sequence designated abcdefg (for review, see Lupas and Gruber 2005). Positions $a$ and $d$, which are usually hydrophobic in character, form the core of the structure and interact with residues in the opposite helix in a "knobs into holes" arrangement (i.e., $a$ and $d$ residues on one helix represent "knobs" that dock into "holes" comprised of a diamond of residues on the other helix). In a canonical parallel homodimer, the equivalent residues in the two helices have the same heptad register, with counterpart $a$ and $d$ side chains intercalating to form alternating " $a$ layers" and "d layers." Thus, there are two homotypic, core-packing layers per heptad.

A striking feature of the BicD structure is that three aromatic residues on each chain are located at the dimer interface (Fig. 2A,B). These are F684 (which, as described above, is mutated to isoleucine in the classical dominant allele), F691, and Y698. Despite their hydrophobic characteristics, aromatic residues are rare at core positions of coiled coils (Lupas et al. 1991; Walshaw and Woolfson
2001). This is presumably because their bulky side chains can cause significant steric clashes at the interhelical interface (Brown 2006). The presence of the aromatic residues in the BicD CC3 homodimer is accommodated by a large offset in their axial positions between the two helices (Fig. 2A,B). Thus, steric hindrance is minimized by the aromatic side chains occupying staggered positions at the core of the structure. The arrangement of the aromatic rings of F684, F691, and Y698 with respect to their counterparts on the other chain strongly suggests the existence of aromatic-aromatic interactions between these side chains (Fig. 2A,B; Supplemental Fig. S3D). These interactions are likely to play an important role in stabilizing the asymmetric coiled-coil structure.

We next analyzed the structure using the SOCKET algorithm (Walshaw and Woolfson 2001), which automatically recognizes knobs into holes interactions and assigns heptad registers within coiled coils. The output of this analysis, which was validated by manual interrogation of the structure, revealed that the offset arrangement of core residues between the two helices extends to the entire N-terminal segment of the coiled coil (Fig. 2C,D). This extensive asymmetric packing necessitates a large difference in the degree of supercoiling of the two helices but nevertheless achieves knobs into holes packing in most layers. Between the most $\mathrm{N}$-terminal packing $a$ layer 


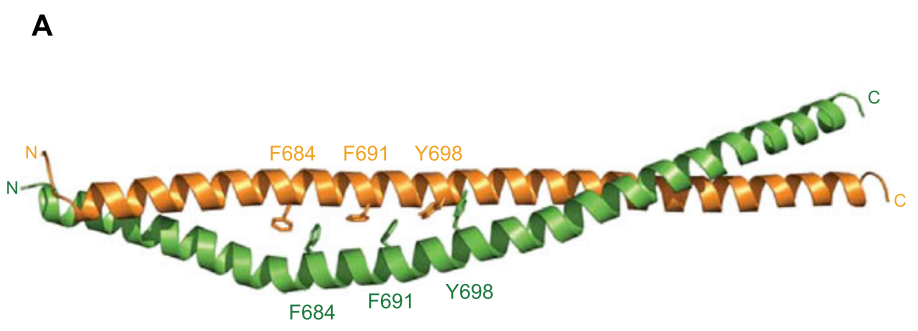

B

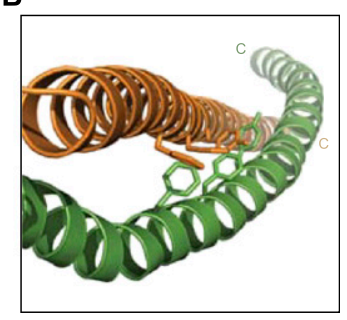

C

a layers:

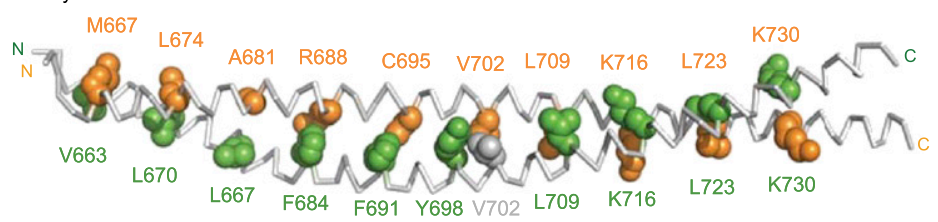

D

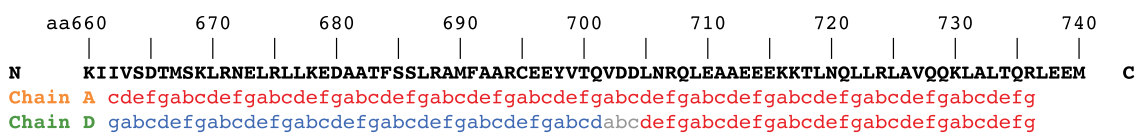

Figure 2. Aromatic residues at the BicD dimer interface are associated with coiled-coil irregularities, including different heptad registers simultaneously adopted by the two chains. $(A, B)$ Positions of aromatic residues at the core of the BicD dimer structure. The arrangement of the six aromatic residues at one side of the dimer suggests the existence of aromatic-aromatic interactions (see also Supplemental Fig. S3D). (C) Residues that form $a$ layers in the structure, as determined by SOCKET and manual inspection. $a$ layers C-terminal to V702 consist of the same residues on the two different chains. $a$ layers $\mathrm{N}$-terminal to V702 are formed by residues that are offset by four positions in the primary sequences of the different chains. Gray residues in $C$ and $D$ had their heptad positions reassigned from the SOCKET output based on manual analysis of the structure. V702 in chain A makes noncomplementary (i.e., not knobs into holes) interactions with both Y698 and V702 in chain D. (D) Heptad positions of all residues determined using SOCKET and manual analysis. Chain A has a continuous register throughout (depicted in red), while chain D has a transition in register (depicted in blue and red).

(formed by M667 of one helix and V663 of the other) and an $a$ layer formed between C695 and F691 on the respective helices, SOCKET identifies nine layers of knobs into holes interactions that are offset by four residue positions (i.e., a "half-heptad") in each chain (Supplemental Tables S1, S2). Thus, the equivalent residues in the two chains simultaneously assume different heptad positions in this region, thereby forming heterotypic "in-plane" layers. Searches of the $\mathrm{CC}^{+}$database (Testa et al. 2009) indicated that this "dual register" feature is unprecedented in parallel, homodimeric coiled coils.

In the locality of the Y698 residues in each chain, layers are no longer formed in-plane but are staggered (Fig. 2C), and complementary knobs into holes interactions are not formed (Supplemental Tables S1,S2). This region represents a transitional zone between the $\mathrm{N}$-terminal region with its in-plane heterotypic cores and the C-terminal segment in which the axial offset of the two helices gradually reduces to zero (Supplemental Fig. S4) and inplane symmetric layers occur (Fig. 2C,D). Thus, from L705 to L733, many homotypic knobs into holes interactions are formed between the two helices (Supplemental Tables S1, S2), even though one helix still exhibits more overall supercoiling than the other.
Identification of a Rabb ${ }^{G T P}$-binding region within the homotypic coiled-coil segment

To determine which features of the BicD structure contribute to cargo recognition, we initially focused on the interaction with Rab6 GTP. First, chemical cross-linking of purified complexes of recombinant $\mathrm{BicD}^{656-745}$ and Rab6 ${ }^{\mathrm{GTP}}$ was performed with nondeuterated and deuterated versions of bis[sulfosuccinimidyl]suberate $\left(\mathrm{BS}^{3}\right.$; a bifunctional, amine-reactive molecule with an $11.4 \AA$ spacer arm) (Sinz 2003). Following proteolytic digestion and mass spectrometry analysis, a single cross-linked peptide species containing sequences from both Rab6 and BicD was detected. Here, K12 of Rab6 was linked to K730 of BicD (Fig. 3A; Supplemental Fig. S5). This finding suggests that these residues are in close proximity in the complex.

Next, the minimal region of BicD that can bind Rab6 ${ }^{\text {GTP }}$ was delineated by performing gel filtration of mixtures of recombinant proteins (Fig. $\left.3 \mathrm{~B}, \mathrm{~B}^{\prime}\right)$. $\mathrm{BicD}^{702-743}$ was the shortest protein tested that was sufficient for robust $\mathrm{Rab6}^{\mathrm{GTP}}$ binding. Interactions of $\mathrm{BicD}^{699-739}$ and $\mathrm{BicD}^{711-745}$ with Rab6 ${ }^{\text {GTP }}$ were barely detectable, even though the gel filtration profiles indicated that the BicD proteins still form dimers. The residues present in $\mathrm{BicD}^{702-743}$ but absent in $\mathrm{BicD}^{699-739}$ and $\mathrm{BicD}^{711-745}$ must either partici- 
A

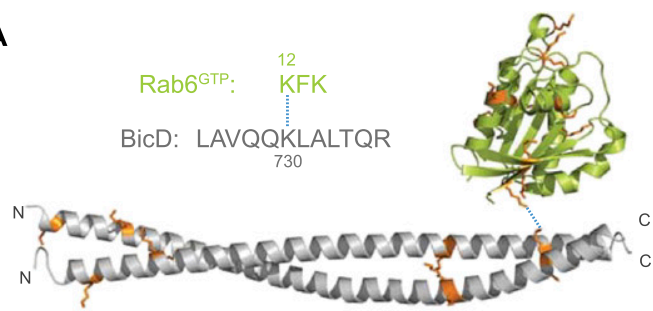

B

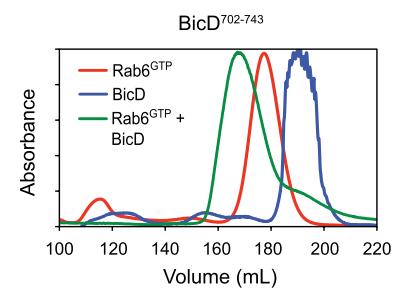

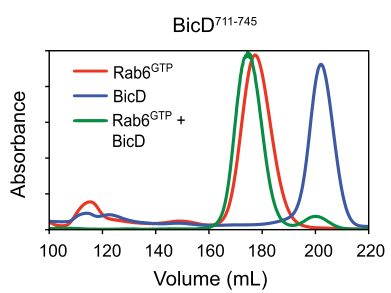

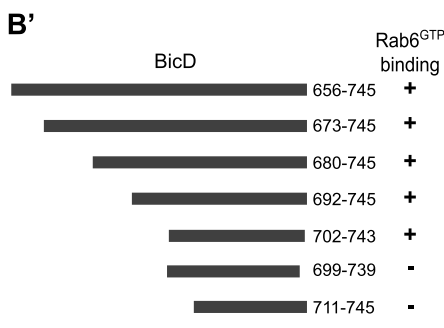

C
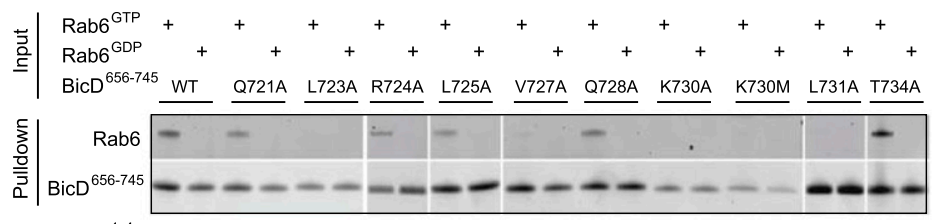

$C^{\prime}$

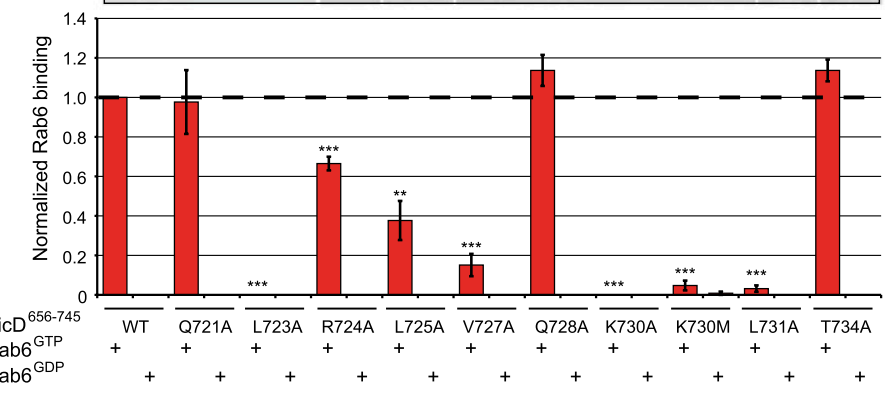

D

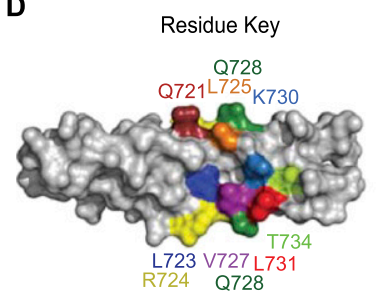

Impact on binding

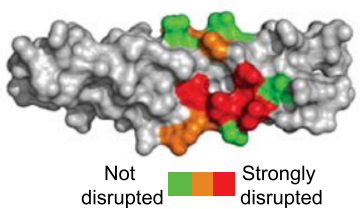

Surface potential

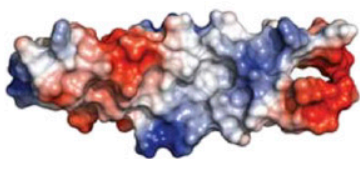

Figure 3. Identification of critical features within $\mathrm{BicD}^{656-745}$ for Rab6 ${ }^{\mathrm{GTP}}$ binding. (A) Sequence of cross-linked peptide identified between $\mathrm{Rab6}^{\mathrm{GTP}}$ and BicD ${ }^{656-745}$ (see Supplemental Fig. S5 for mass spectrum). Blue dashed line indicates cross-linked residues. Lysines within the two proteins that could potentially react with the cross-linker are shown in orange on the crystal structures (Drosophila Rab6 bound to the GTP analog GMP-PNP; PDB: 2Y8E [Walden et al. 2011]); the Rab6 and BicD ${ }^{656-745}$ structures are positioned arbitrarily within the maximal radius of interaction between the cross-linked amine groups of the lysines, which is defined by the $11.4 \AA$ spacer length of the cross-linker. For simplicity, only one copy of Rab6 ${ }^{\mathrm{GTP}}$ is shown. $\left(B, B^{\prime}\right)$ Mapping of the minimal binding fragment for Rab6 ${ }^{\text {GTP }}$ within BicD ${ }^{656-745}$ by gel filtration (1.5:1 molar ratio). Representative elution profiles are shown in $B$. Absorbance values were scaled to correct for differences in molar extinction coefficients of proteins. Binding when the two proteins are mixed results in a substantive leftward shift in the fraction with peak absorbance compared with when either protein is present alone. $\left(B^{\prime}\right)$ Summary of truncation analysis. (+) Robust binding; $(-)$ no or very weak binding. $\left(C, C^{\prime}\right)$ Representative SYPRO Ruby-stained gels $(C)$ and quantification $\left(C^{\prime}\right)$ from pull-down experiments assessing binding of Rab6 ${ }^{\mathrm{GTP}}$ and Rab6 ${ }^{\mathrm{GDP}}$ to His::BicD ${ }^{656-745}$ variants immobilized on a nickel affinity matrix. Mean values for Rab6 binding (generated following correction for variation in BicD signal in each sample and normalized to values for Rab6 ${ }^{\mathrm{GTP}}$ binding to wild-type [WT] His::BicD ${ }^{656-745}$ ) derive from six experiments. Error bars are SEM. $\left(^{\star \star}\right) P<0.01 ;\left(^{\star \star \star}\right) P<0.001$ (one-sample $t$-tests compared with wild-type His::BicD ${ }^{656-745}$, with $P$-values adjusted for multiple comparisons with the Holm-Bonferroni method). Rab6 proteins were GST-tagged. $(D)$ Surface representations of BicD ${ }^{702-743}$ showing positions of residues mutated for binding assays (left), impact of mutations on normalized Rab6 ${ }^{\text {GTP }}$ binding ([red] strongly disrupted, $0.0-0.25$; [orange] partially disrupted, $0.25-0.75$; [green] not disrupted, $>0.75$ ) (center), and electrostatic potential (coded as in Fig. 1C) (right). 
pate in Rab6 ${ }^{\mathrm{GTP}}$ recognition directly or have an indirect role in maintaining the conformation of the binding site within the coiled coil. This latter scenario has been observed in studies of the Sec $2 p$ coiled coil, in which substantial flanking stretches are needed to form the short binding interface for the Sec4p Rab protein (Dong et al. 2007; Sato et al. 2007a).

The data presented above reveal that Rab6 ${ }^{\mathrm{GTP}}$ associates with the region of $\mathrm{BicD}^{656-745}$ that forms a homotypic coiled coil and support the significance of the identified cross-link between BicD K730 and Rab6. Remarkably, a K730M mutation is one of a small number of BicD-null alleles found in genetic screens in Drosophila (Ran et al. 1994). This mutation disrupts binding of Drosophila BicD to Rab6 (Dienstbier et al. 2009), and the same effect is seen for the equivalent substitution in mammalian BICD2 and BICDR-1 (Schlager et al. 2010). However, it was not known whether the lysine-to-methionine mutation alters cargo binding indirectly by changing the global structure of BicD. Our identification of a cross-link on K730 indicates that this residue could participate directly in recognition of Rab6 ${ }^{\mathrm{GTP}}$.

We therefore generated a series of mutations in the vicinity of $\mathrm{K} 730$ to assess whether this region of $\mathrm{BicD}$ binds directly to Rab6 ${ }^{\text {GTP }}$ (Fig. 3C, $\left.C^{\prime}, D\right)$. Recombinant wild-type or mutant His::BicD ${ }^{656-745}$ proteins were immobilized on a nickel affinity matrix and incubated with Rab6 $^{\text {GTP }}$ or Rab6 ${ }^{\mathrm{GDP}}$ (Rab6 ${ }^{\mathrm{T} 27 \mathrm{~N}}$ purified in the presence of GDP). As expected, no binding of any BicD variant to Rab6 ${ }^{\mathrm{GDP}}$ was observed (Fig. 3C,C'). Wild-type BicD ${ }^{656-745}$ bound robustly to Rab6 ${ }^{\mathrm{GTP}}$, as did the Q721A, Q728A, and T734A mutants. In contrast, L723A, V727A, K730A, $\mathrm{K} 730 \mathrm{M}$, and L731A dramatically diminished association with Rab6 ${ }^{\text {GTP }}$, whereas R724A and L725A caused a partial, but statistically significant, reduction in binding. The requirement for leucine and valine residues in BicD for binding Rab6 is consistent with the well-established importance of interactions between hydrophobic features on Rab effectors with a hydrophobic triad within the $\mathrm{G}$ protein (Fernandes et al. 2009). Figure 3D summarizes the findings from the mutagenesis experiments in the context of the BicD structure, with the side chain positions of residues important for Rab6 ${ }^{\mathrm{GTP}}$ binding shown in Supplemental Figure S6.

Collectively, our data demonstrate that there is a Rab6 ${ }^{\mathrm{GTP}}$-binding site between residues 702 and 743 of $\mathrm{BicD}$ and suggest that some of the residues between 723 and 731 (inclusive) directly contact the G protein. The contribution of this latter region to direct binding is also supported by its pronounced conservation within BicD orthologs and paralogs that are known to associate with Rab6 $^{\text {GTP }}$ (Supplemental Fig. S7A; Schlager et al. 2010). Importantly, despite the overall asymmetry of the BicD CC3 structure, the spatial arrangement of the residues important for Rab6 ${ }^{\mathrm{GTP}}$ binding is very similar on the opposing faces of the coiled coil (Supplemental Fig. S7B). This can explain the 2:2 stoichiometry of BicD:Rab6 ${ }^{\text {GTP }}$ binding (Fig. 1A,B); i.e., with one Rab6 ${ }^{\text {GTP }}$ able to interact with each copy of the binding site on the opposing faces of the BicD dimer.
Evidence for a common binding site for Egl and $\mathrm{Rab6}^{\mathrm{GTP}}$ on BicD CC3

To investigate whether BicD uses distinct or overlapping sites to bind different cargo adaptors, we turned our attention to its interaction with Egl. This is a 1004-aminoacid RNA-binding protein that binds features within BicD $^{647-782}$ in order to link RNA localization signals to dynein (Dienstbier et al. 2009). Because association of Egl is disrupted by $\mathrm{BicD}^{\mathrm{K} 730 \mathrm{M}}$ in vitro and in vivo (Dienstbier et al. 2009), Egl and Rab6 might recognize overlapping features on BicD. To test this possibility, we translated in vitro the first 79 residues of Drosophila Egl, which are necessary and sufficient for specific binding to BicD (Dienstbier et al. 2009), and assessed binding to truncations of CC3 using pull-down assays. Egl ${ }^{1-79}$ bound to proteins containing BicD residues 702-743 (Fig. 4A). In contrast, residues 711-745 were not sufficient for robust binding (Fig. 4A). This behavior is identical to that observed for Rab6 GTP (Fig. 3B, $\mathrm{B}^{\prime}$ ). We also found that all of the other amino acid substitutions in $\mathrm{BicD}^{656-745}$ that inhibited binding to Rab6 ${ }^{\mathrm{GTP}}$ (L723A, R724A, L725A, V727A, and L731A) (Fig. 3C, $C^{\prime}$ ) also strongly inhibited binding to Egl (Fig. 4B). R724A and L725A had substantially stronger effects on Egl binding than on Rab6 ${ }^{\mathrm{GTP}}$ binding, suggesting differences in the relative contributions of at least some BicD residues to the interaction with each partner. The Q721A and T734A BicD mutations, which did not interfere with Rab6 ${ }^{\mathrm{GTP}}$ binding (Fig. 3C, $\mathrm{C}^{\prime}$ ), did not reduce the interaction with Egl (Fig. 4B). Collectively, our data strongly suggest that an identical or very similar site on BicD mediates recognition of both Rab6 GTP and Egl. This notion was corroborated by our finding that Rab6 ${ }^{\text {GTP }}$, but not Rab6 ${ }^{\mathrm{GDP}}$, was able to compete with Egl for the interaction with $\mathrm{BicD}^{656-745}$ in vitro (Fig. 4C; Supplemental Fig. S7C).

\section{Evidence that binding of cargo to BicD promotes recruitment of the dynein motor}

As described above, it is not known whether the binding of cargo to $\mathrm{BicD}$ is required for the recruitment or activation of the dynein motor. To address this issue, we sought to compare the ability of wild-type and L731A fulllength BicD to be transported by, and interact with, dynein in vivo. L731A strongly inhibits binding of both Rab6 ${ }^{\mathrm{GTP}}$ and $\mathrm{Egl}$ to $\mathrm{CC} 3$ in vitro (Figs. 3C, $\mathrm{C}^{\prime}, 4 \mathrm{~B}$ ). Furthermore, because the side chain of L731 (which occupies a $b$ heptad position) is exposed to the solvent (Supplemental Fig. S6), the interpretation of the consequences of this mutation is unlikely to be confounded by secondary effects on the overall structure of the dimer interface. Indeed, dimerization of the L731A mutant of BicD ${ }^{656-745}$ was confirmed by SEC-MALS (Supplemental Fig. S7D).

In order to study the BicD-dynein interaction in a physiological context, we generated transgenic flies expressing full-length BicD::GFP with the L731A mutation $\left(\mathrm{BicD}^{\mathrm{L} 731 \mathrm{~A}}:: \mathrm{GFP}\right)$ under the control of a ubiquitous promoter. Whereas a wild-type BicD::GFP transgene $\left(\right.$ BicD $^{\mathrm{WT}}:$ :GFP) (Dienstbier et al. 2009) was able to efficiently restore the ability of BicD zygotic-null mutants to 
Liu et al.

A
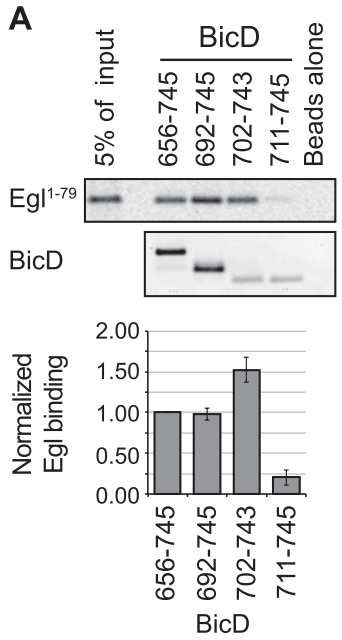

B
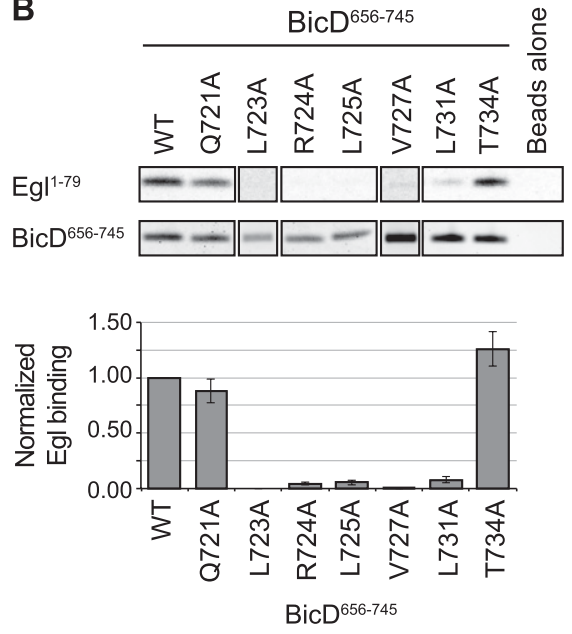

Figure 4. The Egl-binding site on BicD is highly similar to, or the same as, the binding site for Rab6 ${ }^{\mathrm{GTP}}$. $(A, B)$ Representative data (top) and quantification (bottom) of truncation $(A)$ and mutational $(B)$ analysis of the features of BicD important for binding Egl. Egl ${ }^{1-79}$ was translated in vitro in the presence of ${ }^{35} \mathrm{~S}$-methionine and incubated with His-tagged BicD proteins immobilized on a nickel affinity matrix. Mean values for $\mathrm{Egl}^{1-79}$ binding (generated following correction for variation in BicD signal in each sample and normalized to values for $\mathrm{Egl}^{1-79}$ binding to wild-type [WT] His::BicD ${ }^{656-745}$ ) derive from three experiments for each condition, except for wild type and L731A (six each). Error bars are SEM. (C) Immunoblots from a representative pull-down assay showing that the binding of His::BicD ${ }^{656-745}$ to recombinant GST::Egl ${ }^{1-79}$ immobilized on a glutathione sepharose matrix is reduced by increasing concentrations of Rab6 ${ }^{\mathrm{GTP}}$. $\mathrm{BicD}^{656-745}$ does not bind to GST alone. Rab6 ${ }^{\mathrm{GDP}}$ does not compete for Egl:BicD binding even at an eightfold molar excess to BicD (Supplemental Fig. S7C). Proteins were visualized with antibodies against His or GST.

eclose from the pupal case, this was not the case for transgenes expressing similar levels of $\mathrm{BicD}^{\mathrm{L} 731 \mathrm{~A}}:$ :GFP (Fig. 5A; Supplemental Fig. S8A). BicD ${ }^{\mathrm{L} 731 \mathrm{~A}}:$ :GFP did, however, give a partial suppression of the lethal phenotype (Fig. 5A). This is consistent with some residual binding of L731A BicD ${ }^{656-745}$ to both Rab6 ${ }^{\mathrm{GTP}}$ and $\mathrm{Egl}$ in vitro (Fig. $3 \mathrm{C}^{\prime}, 4 \mathrm{~B}$ ) and the overexpression of the GFP fusion proteins relative to endogenous BicD (see Fig. 5C, input, $\alpha$-BicD|. Nonetheless these data confirm the importance of the region responsible for binding Rab6 ${ }^{\mathrm{GTP}}$ and $\mathrm{Egl}$ in vitro for the function of BicD in vivo.

We next examined the distribution of equivalently expressed $\mathrm{BicD}^{\mathrm{WT}}:: \mathrm{GFP}$ and $\mathrm{BicD}^{\mathrm{L} 731 \mathrm{~A}}:: \mathrm{GFP}$ proteins during early oogenesis. In this well-characterized system, dynein-mediated translocation of BicD-containing complexes results in an enrichment of BicD in the oocyte (where minus ends of microtubules concentrate) compared with the interconnected nurse cells (Wharton and Struhl 1989; Theurkauf et al. 1993; Navarro et al. 2004). Unlike BicD ${ }^{\mathrm{WT}}:$ :GFP, the L731A mutant protein was not enriched in the oocyte compared with the nurse cells (Fig. $5 \mathrm{~B})$. Hence, there is a correlation between the capacity of BicD to efficiently bind cargo adaptors and its ability to undergo dynein-dependent trafficking.

Diminished dynein-dependent transport of $\mathrm{BicD}^{\mathrm{L} 731 \mathrm{~A}}:$ : GFP could be due to reduced association with the dynein complex or because dynein is recruited at normal levels but is not active. To discriminate between these possibilities, we used a GFP-binding protein (GBP) affinity matrix to pull down $B^{-1 c D^{W T}}:: G F P$ or BicD ${ }^{\mathrm{L} 731 \mathrm{~A}}:: \mathrm{GFP}$ from embryo extracts. As expected, the L731A mutation strongly reduced, but did not abolish, the ability of full-length BicD to associate with a cargo adaptor in vivo, as assessed by immunoblotting the precipitates for endogenous Egl (note that specific antibodies to Drosophila Rab6 are not available) (Fig. 5C). Strikingly, L731A also strongly reduced the amount of dynein precipitated with BicD::GFP, as judged by immunoblotting for the dynein heavy chain (Dhc) and dynein intermediate chain (Dic) subunits (Fig. 5C). These data provide evidence that binding of cargo adaptors to $\mathrm{CC} 3$ promotes the ability of more $\mathrm{N}$-terminal sequences of BicD to complex with dynein.

\section{Features in the heterotypic coiled-coil region regulate dynein binding to BicD}

As described above, the $\mathrm{F} 684$ residue of $\mathrm{BicD}$, which is mutated to isoleucine in the classical dominant allele $B i c D^{1}$ (Wharton and Struhl 1989), is one of three aromatic residues on each helix that, very unusually, are located at the core of the dimer. It appears that this feature plays an important role in the heterotypic core-packing arrangement of the $\mathrm{N}$-terminal region of the structure. To investigate the function of this heterotypic region, we assayed the consequence of the F684I mutation on the interactions of BicD with its binding partners. Previous analysis of classical BicD dominant mutations in Drosophila indicated that the anterior-posterior patterning defects are due to an increase in Egl- and dynein-mediated anterior localization of osk mRNA (Mohler and Wieschaus 1986; Ephrussi et al. 1991; Kim-Ha et al. 1991; Mach and Lehmann 1997; Navarro et al. 2004). This notion was 
A

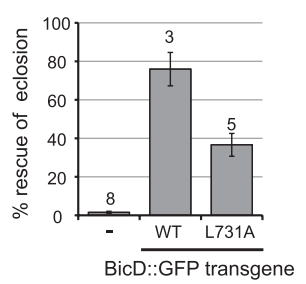

B

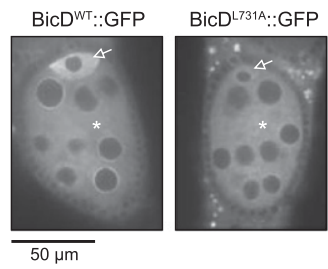

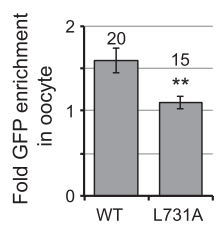

c

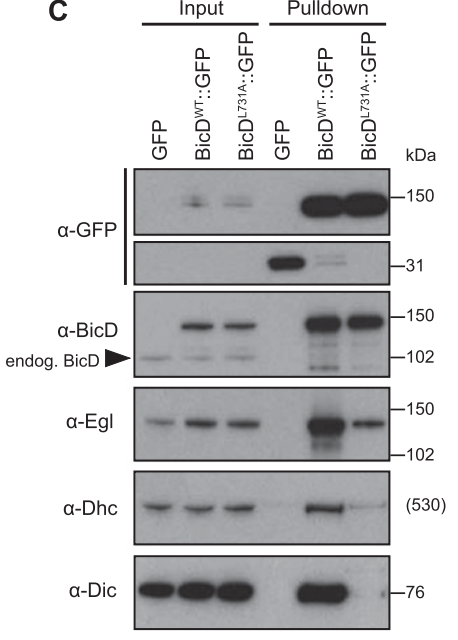

Figure 5. Disruption of the cargo-binding capacity of BicD inhibits dynein recruitment in vivo. $(A)$ Quantification of the ability of BicD::GFP transgenes to rescue lethality caused by lack of endogenous, zygotically expressed BicD. The $Y$-axis shows the number of flies that eclosed from the pupal case of each genotype, expressed as a percentage of the number expected by Mendelian inheritance (i.e., assuming no lethality). Thus, a transgene that completely rescues lethality caused by the absence of endogenous $B i c D$ would have an expected value of $100 \%$. Number of independent transgenic lines expressing comparable levels of BicD::GFP used to derive each mean value is shown above bars (except for the data for the BicD mutant without transgene expression, where mean values were derived from siblings in each of the BicD ${ }^{\mathrm{WT}}:$ :GFP and $\mathrm{BicD}^{\mathrm{L} 731 \mathrm{~A}}:$ :GFP crosses). Error bars represent SEM in this and other panels. BicD-null genotype is R5/Df(2L)TW119. Eclosed BicD-null flies expressing $B_{i c D^{L 731 A}}:$ GFP had an $\sim 50 \%$ reduction in median life span compared with those expressing BicD ${ }^{\mathrm{WT}}:: \mathrm{GFP}$ (data not shown). (B) The L731A mutation disrupts enrichment of BicD::GFP in the oocyte. Representative images and quantification of relative fluorescence in the oocyte (arrow) and cytoplasm of interconnected nurse cells (asterisk) are shown. Number of egg chambers analyzed for each genotype is shown above the bars. $\left(^{\star \star}\right) P<0.01$ (two-tailed $t$-test). Experiments were performed in the presence of endogenous BicD. $(C)$ Immunoblots of GBP pull-downs from embryo extract showing that, relative to $B_{i c D^{W T}}:: G F P, B_{i c D}{ }^{\mathrm{L} 731 \mathrm{~A}}:: \mathrm{GFP}$ has a strongly reduced capacity to associate with endogenous Egl, Dhc, and Dic. Flies expressing GFP alone were used as a specificity control. Note that we did not detect heterodimerization of BicD::GFP proteins with the endogenous BicD (arrowhead; see also Fig. 6E); this could be due to temporal differences in the synthesis of these proteins, which are expressed from different promoters, or cotranslational assembly of the coiled coil.

corroborated by our finding that overexpression of Egl in the female germline in a wild-type BicD background gave rise to a substantial proportion of bicaudal embryos (Supplemental Fig. S8B-E), which was associated with anterior mislocalization of osk mRNA in earlier development (Supplemental Fig. S8M).

We first considered the possibility that the F684I mutation causes a conformational change within $\mathrm{CC} 3$ that results in the neighboring minimal cargo-binding region (702-743) having increased affinity for Egl. To test this notion, we produced recombinant $\mathrm{BicD}^{656-745}$ with the F684I mutation. This protein exhibited a gel filtration profile similar to the wild type (Supplemental Fig. S9), indicating that dimerization was not disrupted. Interestingly, the extent of binding of in vitro translated $\mathrm{Egl}^{1-79}$ to recombinant His: : BicD ${ }^{656-745}$ was not altered by the F684I mutation (Fig. 6A). The mutation also did not affect the degree of association of Rab6 ${ }^{\mathrm{GTP}}$ with $\mathrm{BicD}^{656-745}$ in vitro (Fig. 6B). Thus, F684I does not detectably alter the affinity of isolated CC3 sequences for Egl or Rab6 ${ }^{\mathrm{GTP}}$.

We next hypothesized that F684I increases Egldependent localization of osk mRNA by attenuating the autoinhibitory interaction between CC3 and more $\mathrm{N}$-terminal sequences of BicD (see above). In this manner, the mutation could lead to increased association of osk ribonucleoprotein particles (RNPs) with the dynein motor. To explore this notion, we examined the consequences of the F684I mutation on the ability of BicD to recruit the dynein complex in vivo. Transgenic Drosophila lines were generated expressing full-length $\mathrm{BicD}^{\mathrm{F} 684 \mathrm{I}}:$ GFP ubiquitously. As anticipated, many embryos laid by females expressing this transgene exhibited anterior-posterior pat- terning defects, including a high penetrance of the bicaudal phenotype (Fig. 6C; Supplemental Fig. S8F,G). No such defects were observed when wild-type BicD::GFP was expressed at equivalent levels (Supplemental Fig. S8A,H). We confirmed using in situ hybridization that the patterning defects in the $\mathrm{BicD}^{\mathrm{F} 684 \mathrm{I}}:$ GFP line were associated with anterior mislocalization of osk mRNA in early embryos (Supplemental Fig. S8M). Consistent with this finding, the abnormal patterning of $\mathrm{BicD}^{\mathrm{F} 684 \mathrm{I}}:$ GFP embryos was strongly suppressed by one copy of an osk RNA-null allele (Supplemental Fig. S8I,J).

We observed a striking increase in the ratio of $\mathrm{BicD}^{\mathrm{F} 684 \mathrm{I}}:: \mathrm{GFP}$ in oocytes versus nurse cells compared with that observed for BicD ${ }^{\mathrm{WT}}:: \mathrm{GFP}$ (Fig. 6D). This is compatible with an increase in BicD enrichment in the oocyte reported in an early study of the $B i c D^{1}$ mutation (Wharton and Struhl 1989) and provides additional evidence that the F684I mutation augments dynein-dependent transport.

To test whether the F684I mutation leads to increased dynein-dependent transport by increasing the association of full-length BicD with dynein, we performed GBP pulldowns from extracts of ovaries expressing $B \mathrm{BD}^{\mathrm{WT}}$ ::GFP or BicD ${ }^{\mathrm{F} 6841}$ : $\mathrm{GFP}$. Ovaries were used for these experiments because, unlike embryos, their differentiation was not affected by the expression of $\mathrm{BicD}^{\mathrm{F} 684 \mathrm{I}}:$ GFP. We consistently detected a substantial increase in the amount of dynein precipitated with $\mathrm{BicD}^{\mathrm{F} 684 \mathrm{I}}:: \mathrm{GFP}$ compared with that observed for $\mathrm{BicD}^{\mathrm{WT}}:: \mathrm{GFP}$, as judged by immunoblotting for Dhc (Fig. 6E). Thus, features within the heterotypic coiled-coil region of BicD CC3 normally attenuate the recruitment of dynein mediated by $\mathrm{CCl} / 2$. 
Liu et al.

A

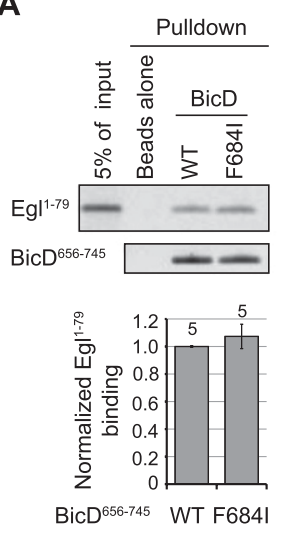

D
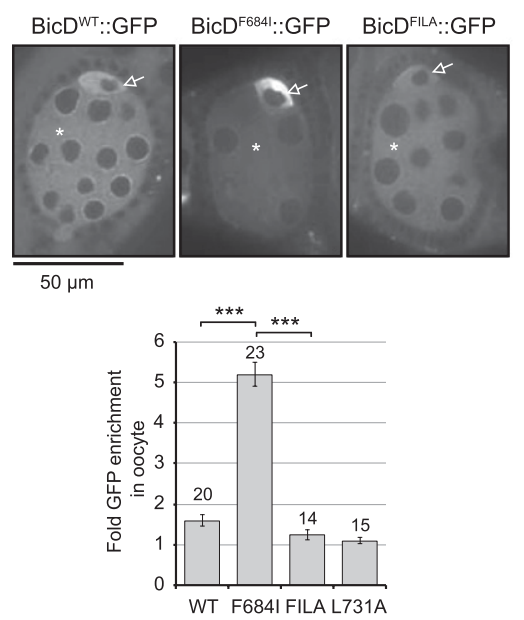

B
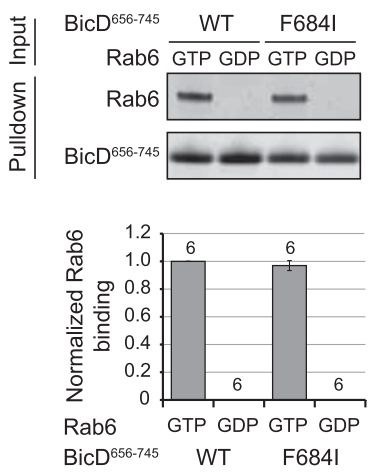

C
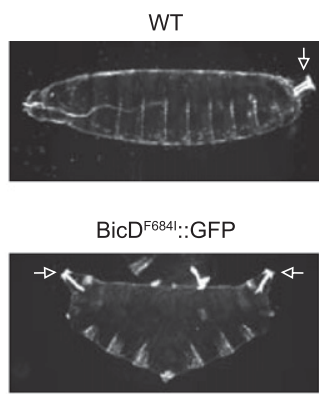

E
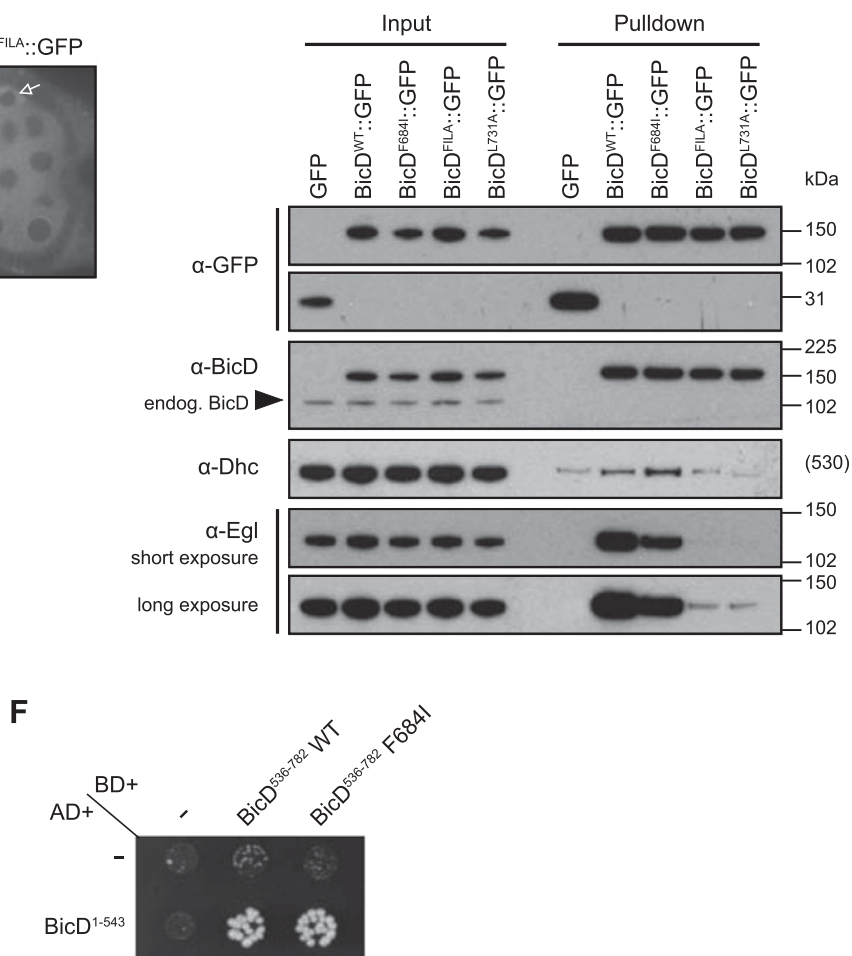

Figure 6. F684I promotes binding of BicD to dynein. $(A, B)$ Representative data $($ top $)$ and quantification $($ bottom) for in vitro binding of wild-type (WT) and F684I BicD ${ }^{656-745}$ to in vitro translated Egl ${ }^{1-79}(A)$ and recombinant GST-tagged Rab6 ${ }^{\mathrm{GTP}}(B)$. In $A$ and $B$, the numbers of experiments performed are shown above the bars. Error bars represent SEM in these and other panels. Assays and quantification for Rab6 and Egl binding were performed as described in the legends for Figures 3 and 4, respectively. (C) Representative cuticle preparations of embryos laid by wild-type (yw strain) and $\operatorname{BicD}^{\mathrm{F} 684 \mathrm{I}}:$ GFP mothers. Posterior structures are duplicated at the expense of anterior structures in the $\mathrm{BicD}^{\mathrm{F} 684 \mathrm{I}}:: \mathrm{GFP}$ preparation; white arrows label telsons (terminal posterior structures). See Supplemental Figure S8 for quantification. $(D)$ Representative images and quantification of relative fluorescence of BicD::GFP proteins in the oocyte (arrow) and cytoplasm of interconnected nurse cells (asterisk). Number of egg chambers analyzed is shown above the bars. $\left({ }^{\star \star \star}\right) P<0.001$ (two-tailed $t$-test). Experiments were performed in the presence of endogenous BicD. The wild-type and L731A data sets in the chart are the same as those in Figure 5B. $(E)$ Immunoblots of GBP pull-downs from ovary extracts assessing the coprecipitation of $\mathrm{Egl}$ and Dhc with BicD::GFP variants. Flies expressing GFP alone were used as a specificity control. The arrowhead indicates the position of endogenous BicD in extracts. There is a 2.7 -fold increase in the Dhe signal in the BicD $^{\mathrm{F} 684 \mathrm{I}}:: \mathrm{GFP}$ sample compared with $\mathrm{BicD}^{\mathrm{WT}}:: \mathrm{GFP}$ (following subtraction of background Dhe signal in the GFP-alone control). Similar or greater increases in Dhe association for $\mathrm{BicD}^{\mathrm{F} 684 \mathrm{I}}:: \mathrm{GFP}$ versus $\mathrm{BicD}^{\mathrm{WT}}:: \mathrm{GFP}$ were seen in a total of five independent experiments. Dhe signals for BicD ${ }^{\mathrm{FILA}}:$ : GFP and BicD ${ }^{\mathrm{L} 731 \mathrm{~A}}:: \mathrm{GFP}$ are comparable with that observed for GFP alone. Note that the association of Dhc with BicD ${ }^{\mathrm{WT}}:: \mathrm{GFP}$ is more readily detected above background levels in embryo extract than ovary extract (cf. Fig. 5C); this could conceivably reflect a lower proportion of autoinhibited BicD in embryos. ( $\alpha$-Egl) Weak binding of Egl to BicD ${ }^{\text {FILA }}::$ GFP and BicD ${ }^{\text {L731A }}::$ GFP can be observed in the longer exposure. $(F)$ Yeast two-hybrid experiment showing that the wild-type and F684I mutant C-terminal regions of BicD both bind to more $\mathrm{N}$-terminal $\mathrm{BicD}$ sequences (indistinguishable rates of growth elicited by the two interactions indicated comparable binding efficiency). Growth was assessed on -HIS plates. (-) GAL4 DNA-binding domain (BD) or activation domain (AD) alone. 
To refine our understanding of how F684I influences the assembly of cargo-motor complexes, we examined whether this mutation affects association of full-length BicD with Egl in vivo. It has previously been shown that full-length $\mathrm{BicD}$ is recruited less efficiently than the isolated C-terminal region to cargos (Hoogenraad et al. 2003; Moorhead et al. 2007; Li et al. 2010). These observations suggest that the cargo-binding features within the C-terminal region of BicD are rendered less accessible by the autoinhibitory interaction with more $\mathrm{N}$-terminal sequences. If the F684I mutation constitutively reduces the autoinhibitory interaction, one would therefore expect increased association of full-length BicD with Egl. However, we did not observe increased precipitation of endogenous Egl with BicD $^{\mathrm{F} 684 \mathrm{I}}:$ :GFP compared with BicD $^{\mathrm{WT}}:$ :GFP (Fig. 6E). In fact, there was consistently a partial reduction in Egl binding to the F684I protein. Thus, the association of BicD with dynein is more limiting than its association with Egl for anterior osk mRNA localization. The reason for reduced association of Egl with the F684I mutant is unclear but could conceivably be associated with an altered conformation of the autoinhibited fulllength protein that reduces accessibility of the Egl-binding site. The notion that F684I does not attenuate BicD's autoinhibitory interaction in the cargo-unbound state was further supported by our observation, using the yeast two hybrid system, that the wild-type and F684I C-terminal third of BicD (containing CC3) bound with indistinguishable efficiency to the remaining $\mathrm{N}$-terminal sequences of the protein (containing CC1 and CC2) (Fig. 6F).

The above observations suggested that the F684I mutation increases the association of BicD with dynein by promoting the release of the autoinhibitory interaction only following cargo binding to the neighboring, homotypic coiled-coil region. If this were the case, one would expect that the F684I mutation does not promote BicD's association with the motor when cargo binding is compromised. We tested this hypothesis by generating transgenic flies expressing a $\mathrm{BicD}^{\mathrm{F} 684 \mathrm{I}+\mathrm{L} 731 \mathrm{~A}}:: \mathrm{GFP}$ double mutant (abbreviated to BicD ${ }^{\text {FILA }:: G F P) . ~ A s ~ a n t i c-~}$ ipated, BicD ${ }^{\text {FILA }:: G F P ~ e x h i b i t e d ~ s t r o n g l y ~ r e d u c e d ~ a s-~}$ sociation with Egl in ovary extract compared with BicD $^{\mathrm{F} 684 \mathrm{I}}:$ :GFP (Fig. 6E) and failed to induce anteriorposterior defects in the vast majority of embryos (Supplemental Fig. S8K). Notably, both the dynein-mediated enrichment of BicD ${ }^{\text {FILA }:: G F P ~ i n ~ t h e ~ o o c y t e ~(F i g . ~ 6 D) ~ a n d ~}$ its biochemical association with Dhc in ovary extract (Fig. 6E) were strongly reduced in comparison with the F684I single mutant. These data therefore provide further evidence that F684I promotes release of BicD autoinhibition in response to cargo binding.

\section{Discussion}

We combined X-ray crystallography with in vitro and in vivo analysis of protein:protein interactions to shed light on mechanisms important for the linkage of cargos to dynein. We revealed that CC3 of the dynein adaptor BicD is a parallel homodimer that exhibits a remarkable degree of axial asymmetry, including regions of homotypic and heterotypic core packing. There is experimental evidence that some parallel, homodimeric coiled coils can exist in alternative homotypic heptad registries (e.g., see Croasdale et al. 2011; Macheboeuf et al. 2011). However, to our knowledge, the extended heterotypic pairing observed in BicD CC3, in which the two chains simultaneously adopt different registers, is unprecedented in parallel homodimers. Our study therefore extends the understanding of the possible architectures of coiled coils. We show that the region of BicD CC3 containing canonical knobs into holes interactions mediates direct binding to different cargo adaptors, with features within the heterotypic segment regulating the recruitment of dynein to more $\mathrm{N}$-terminal sequences of BicD. We discuss below the implications for how different BicD cargos are sorted in vivo and possible mechanisms by which the CC3 domain coordinates cargo binding and motor recruitment.

\section{Implications of a shared binding site for Rab6 GTP and Egl on BicD}

Our study reveals an equivalent minimal binding region on BicD CC3 for Rab6 GTP and Egl, with the same mutations between residues 723 and 731 (inclusive) inhibiting association of both proteins. Thus, these two proteins, which are the two best-characterized cargo adaptors for BicD, recognize the same or highly similar features within the region with homotypic knobs into holes interactions. $\mathrm{Egl}^{1-79}$ and Rab6 do not have primary sequence similarity, suggesting that they share structural features that mediate binding to BicD.

BicD CC3 has been strongly implicated in binding a variety of other proteins (including a pathogen-associated factor) that associate with dynein (Supplemental Fig. S1). The features that we reveal here to be important for Rab6 ${ }^{\mathrm{GTP}}$ and Egl binding are strong candidates to mediate interactions of CC3 with other partners. Consistent with this notion, the $\mathrm{K} 730 \mathrm{M}$ mutation was previously found to disrupt the association of Fragile $\mathrm{X}$ mental retardation protein with BicD (Bianco et al. 2010).

At least some long coiled-coil proteins involved in intracellular sorting have nonoverlapping binding sites for different interactors, presumably allowing coordinated trafficking of proteins with related or cooperative functions (e.g., see Shiba et al. 2006; Rosing et al. 2007; Schweizer Burguete et al. 2008; Sinka et al. 2008). The fact that BicD has not evolved different binding sites for Rab6 ${ }^{\mathrm{GTP}}$ and Egl may be related to the distinct fates of their respective consignments: Golgi-derived vesicles and mRNAs. Egl-associated mRNAs are rapidly delivered to the minus ends of microtubules by bidirectional transport in which minus end-directed motion is much more frequent than plus end-directed motion (Bullock et al. 2006). In contrast, Rab6-positive vesicles have a more uniform cellular distribution, with dynein-based minus end-directed motion counterbalanced by frequent switching to kinesin-driven plus end motion (Matanis et al. 2002). Significant cotransport of Egl-associated and Rab6-associated cargos, which could be afforded by nonoverlapping binding sites on BicD, would therefore be expected to 
impinge on their differential sorting. It is conceivable that some cotransport could occur due to each of the two copies of the binding site on a single BicD molecule being occupied simultaneously by the two different cargo adaptors. However, we did not detect any recruitment of Rab6 to Egl-associated mRNA particles in the Drosophila embryo (Supplemental Fig. S10). Clustering of Egl and Rab6 ${ }^{\mathrm{GTP}}$ molecules on their respective consignments may increase the likelihood that a BicD dimer is occupied exclusively by a single cargo adaptor species.

\section{Insights into the coordination between cargo binding} to BicD and dynein recruitment

As described above, there is substantial evidence that CC3 exerts an inhibitory effect on the ability of more $\mathrm{N}$-terminal BicD sequences to associate with the dynein complex. However, it was not known whether CC3 moderates the interaction of BicD with dynein in both the cargo-bound and -unbound states or whether cargo binding plays an active role in recruitment of the motor complex. We found that mutation of a solvent-exposed $\mathrm{CC} 3$ residue important for efficient binding of BicD to $\mathrm{Rab}^{\mathrm{GTP}}$ and Egl strongly reduces the ability of full-length $\mathrm{BicD}$ to complex with dynein in vivo. These data provide evidence that occupancy of BicD's cargo adaptor-binding site promotes the association of $\mathrm{BicD} \mathrm{CCl} / 2$ with dynein. This process would help coordinate assembly of transport complexes by coupling the availability of cargo to the ability of BicD to bind the motor.

Our analysis of the classical dominant F684I BicD mutation, together with the crystal structure of the wildtype $\mathrm{CC} 3$, provides insights into the features of BicD that regulate binding to dynein in response to cargo. F684 is one of three aromatic residues on each chain of CC3 that appear to play an integral part in the heterotypic corepacking arrangement. We show that F684I does not increase the binding of isolated CC3 sequences to either Egl or Rab6 ${ }^{\mathrm{GTP}}$. Instead, this mutation leads to increased association of $\mathrm{BicD}$ with dynein. Thus, features within the heterotypic coiled-coil segment of BicD CC3 negatively regulate the recruitment of dynein that is mediated by $\mathrm{CCl} / 2$. F691 is also likely to contribute to this process, since our preliminary analysis of transgenic flies expressing full-length $\mathrm{BicD}^{\mathrm{F} 691 \mathrm{II}}:$ GFP reveals a substantial proportion of embryos with anterior-posterior patterning defects (Supplemental Fig. S8L).

In addition to providing insights into the molecular mechanisms that control recruitment of dynein to cargo (see below), our findings offer a long sought after molecular explanation for how the classical F684I mutation leads to ectopic anterior localization of part of the osk mRNA pool. Our data suggest that the association of the dynein motor with Egl/BicD-associated osk RNPs (a process that plays a physiological role in the delivery of osk from the nurse cells to the oocyte [Navarro et al. 2004; Clark et al. 2007]) is increased by the mutation, thereby opposing the plus end-directed, kinesin-1-driven transport of the mRNA to the posterior cortex that begins during mid-oogenesis (Brendza et al. 2000; Navarro et al. 2004; Zimyanin et al. 2008).
How could the F684 residue of CC3 influence dynein binding mediated by more N-terminal regions? The most parsimonious explanation is that this residue participates in or influences the autoinhibitory interaction between CC3 and $\mathrm{CC} 1 / 2$. As described in the results, the F684I mutation does not abrogate autoinhibition in the absence of cargo binding. Instead, this mutation appears to promote the relief of autoinhibition once cargo is bound. Thus, our data suggest a model in which F684 forms an important part of a "molecular switch" that stimulates release of the autoinhibitory interaction following cargo binding to the homotypic coiled-coil region (Fig. 7). The F684I mutation may increase the sensitivity of this switch such that adoption of a dynein-competent state of $\mathrm{BicD}$ is achieved much more readily upon cargo binding than in the wild-type situation.

In structural terms, the replacement of the phenylalanine that participates in the interchain aromatic-aromatic interactions with the $\beta$-branched isoleucine side chain is likely to destabilize the heterotypic arrangement of core packing. It is therefore tempting to speculate that, in the case of wild-type BicD, binding of cargo adaptors to the homotypic coiled-coil region stimulates a structural rearrangement in the neighboring, heterotypic region of $\mathrm{CC} 3$ that releases the autoinhibitory interaction with $\mathrm{CC} 1 / 2$. Previous studies of coiled coils have provided evidence for long-distance allosteric changes (e.g., see Brown et al. 2001; Laughton et al. 2008; Carter 2013). In the case of BicD, one exciting scenario is that the release of autoinhibition upon cargo binding involves a transition between heterotypic and homotypic core packing through a half-heptad shift in the relative axial position of one of the helices. Long-term

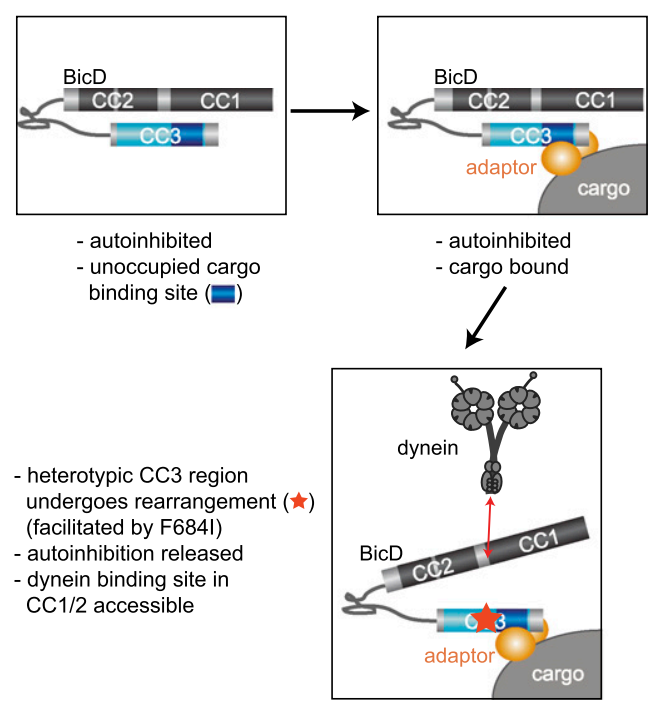

Figure 7. Model for the role of BicD CC3 in orchestrating recruitment of cargo to dynein. See the text for details. Dimeric $\mathrm{BicD}$ is depicted. Note that it is currently not clear which features of BicD participate in the autoinhibitory binding and how this interaction is overcome by cargo association. (Bottom panel) It is also not known whether the proposed release of autoinhibition following cargo binding can also be stimulated by external regulators and/or interactions of the dynein complex with CC1/2. Dynactin is not shown for simplicity. Not to scale. 
studies will be required to test this and other possibilities and shed light on the structural basis of the autoinhibitory interaction.

\section{Materials and methods}

\section{Plasmids}

Constructs encoding wild-type Drosophila BicD were generated from full-length cDNAs by PCR. Constructs for bacterial expression of BicD were produced in the pET28a vector encoding an $\mathrm{N}$-terminal histidine (His) affinity tag followed by the tobacco etch virus (TEV) protease cleavage sequence. For Drosophila P-element-based transgenesis, sequences encoding full-length $\mathrm{BicD}$ were cloned into a pCASPER-based vector with a ubiquitous $\alpha$-tubulin promoter and a cassette encoding a C-terminal GFP tag (Dienstbier et al. 2009). Mutations were generated by QuikChange mutagenesis (Stratagene). Full-length (amino acids 1-208) Drosophila Rab6 ${ }^{\mathrm{Q} 71 \mathrm{~L}}$ (GTP locked form) and Rab6 ${ }^{\mathrm{T} 27 \mathrm{~N}}$ (GDP locked form) were produced from the pGEX-6T-1 vector (which provides an $\mathrm{N}$-terminal GST tag) (constructs kindly provided by A. Gillingham, MRC Laboratory of Molecular Biology). Where indicated, sequences encoding Rab6 ${ }^{\mathrm{Q} 71 \mathrm{~L}}$ were subcloned into the pET28a vector to produce His-tagged protein. A pGEX-4T-1 vector encoding Drosophila Egl $^{1-79}$ was kindly provided by M. Dienstbier (MRC Laboratory of Molecular Biology). The sequences of all inserts were verified before use.

\section{Crystallization}

The Laboratory of Molecular Biology nanoliter crystallization robotic facility was used for a broad screen of 1440 crystallization conditions. Initial crystals of native $\mathrm{BicD}^{656-745}$ formed in $100-\mathrm{nL}$ sitting drops containing a $1: 1 \mathrm{(v/v)}$ ratio of protein to reservoir solution (condition A11; MORPHEUS, Molecular Dimensions Ltd.). Diffraction-quality crystals of native and SeMet $\mathrm{BicD}^{656-745}$ were obtained at $18^{\circ} \mathrm{C}$ by the hanging-drop approach in an optimized reservoir solution containing 0.1 M Tris (pH 8.5), $5 \%$ PEG4000, $10 \%$ glycerol, $30 \mathrm{mM} \mathrm{MgCl}_{2}$, and $30 \mathrm{mM} \mathrm{CaCl}_{2}$ using $2 \mu \mathrm{L}$ of protein sample and $2 \mu \mathrm{L}$ of reservoir solution. Prior to freezing in liquid nitrogen, crystals were cryoprotected in the same reservoir solution with an elevated concentration of glycerol $(25 \%)$.

Additional Materials and Methods can be found in the Supplemental Material.

\section{Accession number}

The BicD CC3 structure reported here has been deposited in the Research Collaboratory for Structural Bioinformatics (RCSB) Protein Data Bank with the ID code 4BL6.

\section{Acknowledgments}

We are very grateful to the staff of Diamond beamlines I03 and I04 for assistance with data collection, Roger Williams for invaluable help with the structure determination, Robert Nicholls and Garib Murshudov for help with Supplemental Figure S7B, and Julien Compagnon and Antoine Guichet for initial assistance with the experiments documented in Supplemental Figure S10. We also thank Andrew Carter and Roger Williams for comments on the manuscript, and Florian Boehl, Martin Dienstbier, Alison Gillingham, Ramanujan Hegde, Daniel St Johnston, Ruth Lehmann, Caryn Navarro, and Beat Suter for reagents or advice. This work was supported by UK MRC core funding (U105178790) and a Lister Institute Research Prize (to S.L.B.).

\section{References}

Bergbrede T, Chuky N, Schoebel S, Blankenfeldt W, Geyer M, Fuchs E, Goody RS, Barr F, Alexandrov K. 2009. Biophysical analysis of the interaction of Rab6a GTPase with its effector domains. J Biol Chem 284: 2628-2635.

Bianco A, Dienstbier M, Salter HK, Gatto G, Bullock SL. 2010. Bicaudal-D regulates fragile $X$ mental retardation protein levels, motility, and function during neuronal morphogenesis. Curr Biol 20: 1487-1492.

Brendza RP, Serbus LR, Duffy JB, Saxton WM. 2000. A function for kinesin I in the posterior transport of oskar mRNA and Staufen protein. Science 289: 2120-2122.

Brown JH. 2006. Breaking symmetry in protein dimers: Designs and functions. Protein Sci 15: 1-13.

Brown JH, Kim KH, Jun G, Greenfield NJ, Dominguez R, Volkmann N, Hitchcock-DeGregori SE, Cohen C. 2001. Deciphering the design of the tropomyosin molecule. Proc Natl Acad Sci 98: 8496-8501.

Bullock SL, Nicol A, Gross SP, Zicha D. 2006. Guidance of bidirectional motor complexes by mRNA cargoes through control of dynein number and activity. Curr Biol 16: 14471452.

Carter AP. 2013. Crystal clear insights into how the dynein motor moves. J Cell Sci 126: 705-713.

Clark A, Meignin C, Davis I. 2007. A Dynein-dependent shortcut rapidly delivers axis determination transcripts into the Drosophila oocyte. Development 134: 1955-1965.

Coutelis JB, Ephrussi A. 2007. Rab6 mediates membrane organization and determinant localization during Drosophila oogenesis. Development 134: 1419-1430.

Croasdale R, Ivins FJ, Muskett F, Daviter T, Scott DJ, Hardy T, Smerdon SJ, Fry AM, Pfuhl M. 2011. An undecided coiled coil: The leucine zipper of Nek2 kinase exhibits atypical conformational exchange dynamics. J Biol Chem 286: 2753727547.

Dienstbier M, Boehl F, Li X, Bullock SL. 2009. Egalitarian is a selective RNA-binding protein linking mRNA localization signals to the dynein motor. Genes Dev 23: 1546-1558.

Dong G, Medkova M, Novick P, Reinisch KM. 2007. A catalytic coiled coil: Structural insights into the activation of the Rab GTPase Sec4p by Sec2p. Mol Cell 25: 455-462.

Ephrussi A, Dickinson LK, Lehmann R. 1991. Oskar organizes the germ plasm and directs localization of the posterior determinant nanos. Cell 66: 37-50.

Fernandes H, Franklin E, Recacha R, Houdusse A, Goud B, Khan AR. 2009. Structural aspects of Rab6-effector complexes. Biochem Soc Trans 37: 1037-1041.

Hoogenraad CC, Akhmanova A, Howell SA, Dortland BR, De Zeeuw CI, Willemsen R, Visser P, Grosveld F, Galjart N. 2001. Mammalian Golgi-associated Bicaudal-D2 functions in the dynein-dynactin pathway by interacting with these complexes. EMBO J 20: 4041-4054.

Hoogenraad CC, Wulf P, Schiefermeier N, Stepanova T, Galjart N, Small JV, Grosveld F, de Zeeuw CI, Akhmanova A. 2003. Bicaudal D induces selective dynein-mediated microtubule minus end-directed transport. EMBO I 22: 6004-6015.

Januschke J, Nicolas E, Compagnon J, Formstecher E, Goud B, Guichet A. 2007. Rab6 and the secretory pathway affect oocyte polarity in Drosophila. Development 134: 34193425.

Kardon JR, Vale RD. 2009. Regulators of the cytoplasmic dynein motor. Nat Rev Mol Cell Biol 10: 854-865.

Kim-Ha J, Smith JL, Macdonald PM. 1991. oskar mRNA is localized to the posterior pole of the Drosophila oocyte. Cell 66: 23-35. 
Liu et al.

Laughton CA, Luisi BF, Pratap JV, Calladine CR. 2008. A potential molecular switch in an $\alpha$-helical coiled coil. Proteins 70: $25-30$.

Li X, Kuromi H, Briggs L, Green DB, Rocha JJ, Sweeney ST, Bullock SL. 2010. Bicaudal-D binds clathrin heavy chain to promote its transport and augments synaptic vesicle recycling. EMBO J 29: 992-1006.

Lupas AN, Gruber M. 2005. The structure of $\alpha$-helical coiled coils. Adv Protein Chem 70: 37-78.

Lupas A, Van Dyke M, Stock J. 1991. Predicting coiled coils from protein sequences. Science 252: 1162-1164.

Mach JM, Lehmann R. 1997. An Egalitarian-BicaudalD complex is essential for oocyte specification and axis determination in Drosophila. Genes Dev 11: 423-435.

Macheboeuf P, Buffalo C, Fu CY, Zinkernagel AS, Cole JN, Johnson JE, Nizet V, Ghosh P. 2011. Streptococcal M1 protein constructs a pathological host fibrinogen network. Nature 472: 64-68.

Matanis T, Akhmanova A, Wulf P, Del Nery E, Weide T, Stepanova T, Galjart N, Grosveld F, Goud B, De Zeeuw CI, et al. 2002. Bicaudal-D regulates COPI-independent Golgi-ER transport by recruiting the dynein-dynactin motor complex. Nat Cell Biol 4: 986-992.

Mohler J, Wieschaus EF. 1986. Dominant maternal-effect mutations of Drosophila melanogaster causing the production of double-abdomen embryos. Genetics 112: 803-822.

Moorhead AR, Rzomp KA, Scidmore MA. 2007. The Rab6 effector Bicaudal D1 associates with Chlamydia trachomatis inclusions in a biovar-specific manner. Infect Immun 75: 781-791.

Navarro C, Puthalakath H, Adams JM, Strasser A, Lehmann R. 2004. Egalitarian binds dynein light chain to establish oocyte polarity and maintain oocyte fate. Nat Cell Biol 6: 427-435.

Ran B, Bopp R, Suter B. 1994. Null alleles reveal novel requirements for Bic-D during Drosophila oogenesis and zygotic development. Development 120: 1233-1242.

Rosing M, Ossendorf E, Rak A, Barnekow A. 2007. Giantin interacts with both the small GTPase Rab6 and Rab1. Exp Cell Res 313: 2318-2325.

Sato Y, Fukai S, Ishitani R, Nureki O. 2007a. Crystal structure of the Sec4p.Sec2p complex in the nucleotide exchanging intermediate state. Proc Natl Acad Sci 104: 8305-8310.

Sato Y, Shirakawa R, Horiuchi H, Dohmae N, Fukai S, Nureki O. 2007b. Asymmetric coiled-coil structure with guanine nucleotide exchange activity. Structure 15: 245-252.

Schlager MA, Kapitein LC, Grigoriev I, Burzynski GM, Wulf PS, Keijzer N, de Graaff E, Fukuda M, Shepherd IT, Akhmanova A, et al. 2010. Pericentrosomal targeting of Rab6 secretory vesicles by Bicaudal-D-related protein 1 (BICDR-1) regulates neuritogenesis. EMBO J 29: 1637-1651.

Schweizer Burguete AS, Fenn TD, Brunger AT, Pfeffer SR. 2008. $\mathrm{Rab}$ and Arl GTPase family members cooperate in the localization of the golgin GCC185. Cell 132: 286-298.

Shiba T, Koga H, Shin HW, Kawasaki M, Kato R, Nakayama K, Wakatsuki S. 2006. Structural basis for Rab11-dependent membrane recruitment of a family of Rab11-interacting protein 3 (FIP3)/Arfophilin-1. Proc Natl Acad Sci 103: 1541615421.

Short B, Preisinger C, Schaletzky J, Kopajtich R, Barr FA. 2002. The Rab6 GTPase regulates recruitment of the dynactin complex to Golgi membranes. Curr Biol 12: 1792-1795.

Sinka R, Gillingham AK, Kondylis V, Munro S. 2008. Golgi coiled-coil proteins contain multiple binding sites for Rab family G proteins. J Cell Biol 183: 607-615.

Sinz A. 2003. Chemical cross-linking and mass spectrometry for mapping three-dimensional structures of proteins and protein complexes. J Mass Spectrom 38: 1225-1237.
Splinter D, Razafsky DS, Schlager MA, Serra-Marques A, Grigoriev I, Demmers J, Keijzer N, Jiang K, Poser I, Hyman $\mathrm{AA}$, et al. 2012. BICD2, dynactin and LIS1 cooperate in regulating dynein recruitment to cellular structures. Mol Biol Cell 23: 4226-4241.

Stuurman N, Haner M, Sasse B, Hubner W, Suter B, Aebi U. 1999. Interactions between coiled-coil proteins: Drosophila lamin Dm0 binds to the bicaudal-D protein. Eur J Cell Biol 78: 278-287.

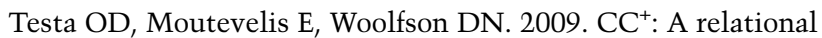
database of coiled-coil structures. Nucleic Acids Res 37: D315-D322.

Theurkauf WE, Alberts BM, Jan YN, Jongens TA. 1993. A central role for microtubules in the differentiation of Drosophila oocytes. Development 118: 1169-1180.

Walden M, Jenkins HT, Edwards TA. 2011. Structure of the Drosophila melanogaster Rab6 GTPase at 1.4 A resolution. Acta Crystallogr Sect F Struct Biol Cryst Commun 67: 744748.

Walshaw J, Woolfson DN. 2001. Socket: A program for identifying and analysing coiled-coil motifs within protein structures. J Mol Biol 307: 1427-1450.

Wharton RP, Struhl G. 1989. Structure of the Drosophila BicaudalD protein and its role in localizing the the posterior determinant nanos. Cell 59: 881-892.

Zimyanin VL, Belaya K, Pecreaux J, Gilchrist MJ, Clark A, Davis I, St Johnston D. 2008. In vivo imaging of oskar mRNA transport reveals the mechanism of posterior localization. Cell 134: 843-853. 


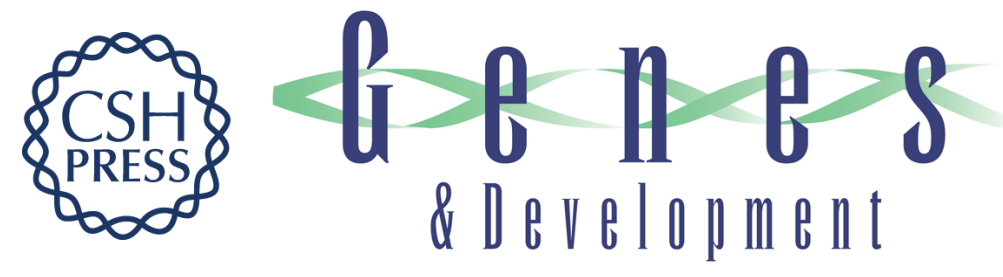

\section{Bicaudal-D uses a parallel, homodimeric coiled coil with heterotypic registry to coordinate recruitment of cargos to dynein}

Yang Liu, Hannah K. Salter, Andrew N. Holding, et al.

Genes Dev. 2013, 27: originally published online May 30, 2013

Access the most recent version at doi:10.1101/gad.212381.112

\section{Supplemental http://genesdev.cshlp.org/content/suppl/2013/05/29/gad.212381.112.DC1 Material}

References This article cites 47 articles, 21 of which can be accessed free at: http://genesdev.cshlp.org/content/27/11/1233.full.html\#ref-list-1

\section{License}

Email Alerting

Receive free email alerts when new articles cite this article - sign up in the box at the top Service

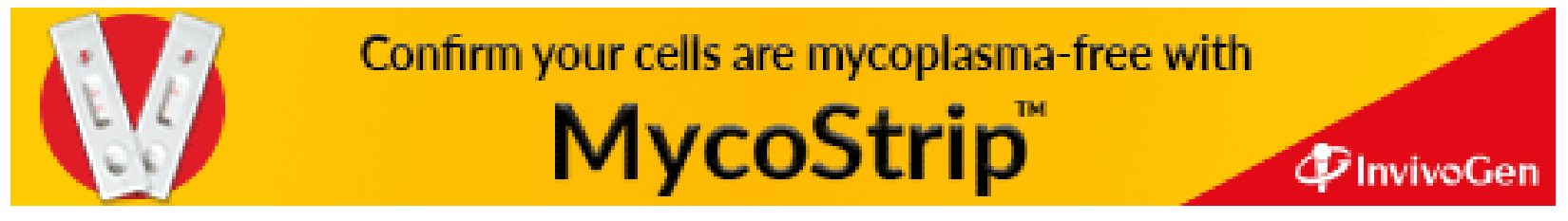

